\title{
On the Radio Occultation Method for Studying Planetary Atmospheres
}

\author{
R. A. Phinney \\ Department of Geological and Geophysical Sciences \\ Princeton University, Princeton, New Jersey 08540 \\ D. L. ANDERSON \\ Seismological Laboratory
California Institute of Technology, Pasadena, California 91109
}

\begin{abstract}
The problem of determining the refractivity profile of a planetary atmosphere from optical or radio occultation data is identical in principle to the problem of determining the variation of seismic velocities in the earth from the observed travel times of seismic body waves. In either case, a complete set of data can be inverted uniquely, the only constraints being those fundamental to geometric optics. Expressions are given for converting observed Doppler shifts to the index of refraction as a function of depth in the atmosphere. The effect of various approximations on the analysis is discussed; it is found that a 'thin atmosphere' approximation simplifies the mathematics and preserves the singularity at the critical ray curvature.
\end{abstract}

\section{INTRODUCTION}

The purpose of this paper is to point out a direct method of transforming radar Doppler data from an occultation experiment directly into a profile of refractive index versus radius. Fjeldbo and Eshleman [1965] discuss in some detail the types of information contained in the phase and amplitude of a radio signal occulted by a planet. Their method of determining the refractive index profile is approximate, but it should give good results without iteration for a planet with a thin atmosphere. They applied their technique to the interpretation of the Mariner 4 occultation experiment on Mars. Cain et al. [1966] analyzed the same data using a model-fitting method, which must assume an analytical description of the model but is not restricted to small bending angles. Kliore et al. [1966], Kliore et al. [1965a, b] Cain et al. [1965], Fjeldbo et al. [1965], and Fjeldbo et al. $[1966 a, b]$ describe the analysis and interpretation of that experiment.

A more general method of analysis is available, based on the Herglotz-Wiechert method for interpreting seismic data. This technique is used to determine velocity-depth profiles in the earth from the observed variation of travel time with arc distance between source and receiver. We describe a form of this theory that is suitable for the radio occultation problem. In the first section, we derive the inversion relations that permit the refractive index profile to be deduced from either the phase shift or the angular path in the atmosphere. In the second section, we show how two kinds of Doppler residuals are used as inputs to the inversion relations. In the following sections, we discuss the analytical form of the ray integrals and review some of the ray-optical constraints on the use of these techniques. For further discussion of such effects as focusing and defocusing, shadow formation, etc., we recommend Bullen's [1963] text.

The geometry of the occultation experiment is summarized in Figure 1. The coordinates are referred to the center of mass of the planet. The refractive index $n$ is a function of $r$ in a shell $R \leq r \leq r_{0}$, where $r_{0}$ is an arbitrary level lying effectively above all the mass of the atmosphere, and $R$ is the radius of the solid planet. The ray path is a straight line outside the atmosphere. It is assumed that the trajectories of the spacecraft and the earth are known with respect to the center of mass of the planet. The indicated spacecraft trajectory is the projection of the actual trajectory in the plane defined by the centers of mass of the spacecraft, earth, and planet. If the properties of the atmosphere depend only on $r$, then only components of ve- 


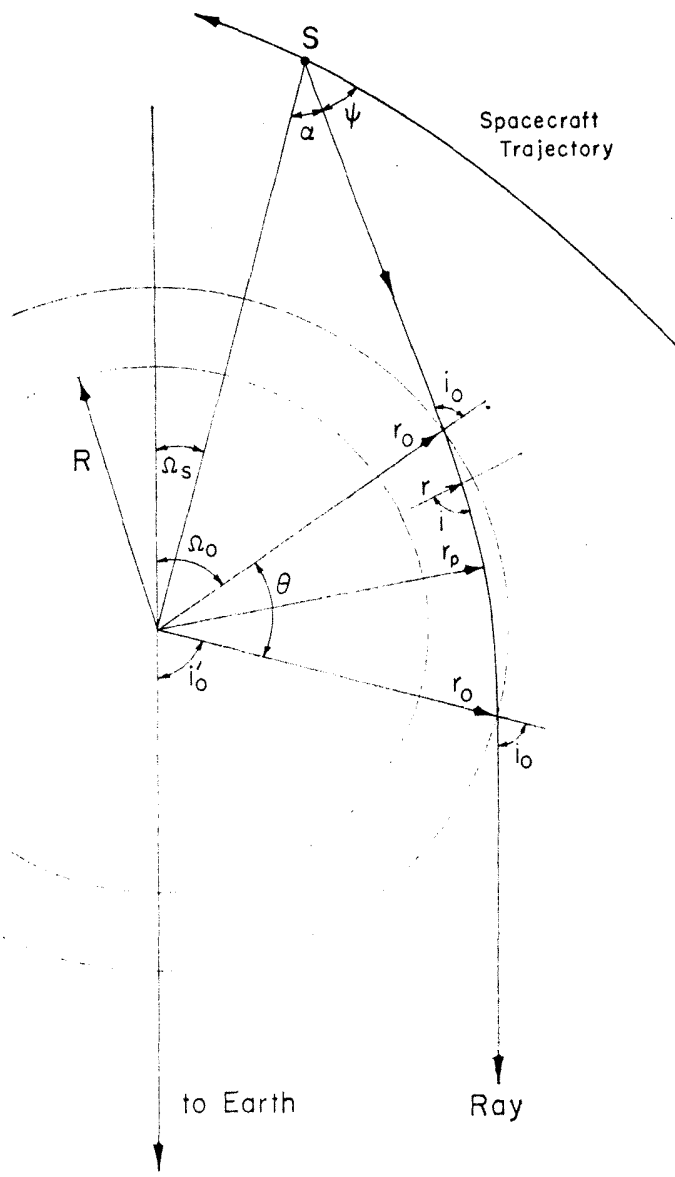

Fig. 1. Geometry of occultation experiment.

locity in the plane of the figure contribute to the observed Doppler shift.

In seismology, the central angle $\theta$ subtended by a ray is observed; the theory is consequently arranged so that the refractive index profile is obtained by operations on $\theta$. In the radiooccultation experiment, the data are obtained in the form of Doppler shift and integrated Doppler phase. The theory given below leads to an expression for the refractive index profile in terms of Doppler residuals. It is shown that two different residual functions are required as input as input to the analysis.

Transformation of $\theta$ and $\Phi$ to a Refractive INdex Profile

A ray parameter $p$ is defined

$$
p=n r \sin i
$$

which is constant along a ray, by Snell's law, and serves as a label of the ray. In the absence of an atmosphere, $p$ would be the radius of closest approach and may be called the impact parameter of the ray. We have immediately:

$$
p=r_{0} \sin i_{0}=r_{p} n_{p}
$$

where the subseript $p$ refers to the turning point of the ray. As an auxiliary variable, we define $\eta$, which varies along a ray, taking the value $r_{0}$ at $r=r_{n}$ and the value $p$ at $r=r_{p}$ as

$$
\eta=n r=p / \sin i
$$

$\eta$ is the silme function of $r$ for all rays and is the value of the impact parameter of the ray, which is horizontal at a given $r$. Integrating along the ray gives the total arc subtended:

$$
\begin{aligned}
\theta(p) & =2 \int_{r_{p}}^{r_{0}} \frac{p}{r}\left(\eta^{2}-p^{2}\right)^{-(1 / 2)} d r \\
& =2 \int_{p}^{r_{0}} \frac{p}{r}\left(\eta^{2}-p^{2}\right)^{-(1 / 2)} \frac{d r}{d \eta} d \eta
\end{aligned}
$$

Likewise, phase shift along the ray (in the atmosphere) is (all angular measures will be in cycles)

$$
\begin{aligned}
\Phi(p) & =2 k_{0} \int_{r}^{r_{0}} \frac{\eta^{2}}{r}\left(\eta^{2}-p^{2}\right)^{-(1 / 2)} d r \\
& =2 k_{0} \int_{p}^{r_{0}} \frac{\eta^{2}}{r}\left(\eta^{2}-p^{2}\right)^{-(1 / 2)} \frac{d r}{d \eta} d \eta
\end{aligned}
$$

Where $k_{\mathrm{n}}=f / c$.

Now set

$$
\begin{aligned}
& y=\eta^{2}-r_{0}{ }^{2} \\
& w=p^{2}-r_{n}{ }^{2}
\end{aligned}
$$

then

$\theta(p)=2 p \int_{w}^{n}(y-w)^{-(1 / 2)} \frac{d \ln r}{d y} d y$

This integral equation for $\ln r(y)$ is in the form of the Abel integral equation and may be solved by reference to the Abel transform pair

$$
f(w)=-K \int_{0}^{w} g^{\prime}(y)(y-w)^{-(1 / 2)} d y
$$

$g(y)=(K \pi)^{-1} \int_{0}^{y} f(w)(w-y)^{-(1 / 2)} d w$

By inspection: 
$\ln \left[r(y) / r_{0}\right]$

$$
=\frac{1}{\pi} \int_{0}^{y} \theta(w) 2 p(w)^{-1}(w-y)^{-(1 / 2)} d w
$$

or

$$
\ln \left[r(\eta) / r_{0}\right]=\frac{1}{\pi} \int_{r_{0}}^{\eta} \frac{\theta(p) d p}{\left(p^{2}-\eta^{2}\right)^{1 / 2}}
$$

The right-hand side is transformed by integration by parts:

$\ln \left[r / r_{0}\right]=\frac{1}{\pi}\left[\theta \cosh ^{-1}(p / \eta)\right]_{r_{0}}^{\eta}$

$$
-\frac{1}{\pi} \int_{r_{0}}^{\eta} \cosh ^{-1}(p / \eta) \frac{d \theta}{d p} d p
$$

The first term on the right-hand side vanishes, and exponentials can be taken:

$$
\begin{aligned}
r(\eta) & =r_{0} \exp \left[-\frac{1}{\pi} \int_{r_{0}}^{\eta} \cosh ^{-1}(p / \eta) \frac{d \theta}{d p} d p\right] \\
& =r_{0} \exp (-M)
\end{aligned}
$$

The integral is always positive; $r_{0}>\eta$, and $d \theta / d p$ is normally negative. It is possible to make $d \theta / d p$ positive over certain ranges of $p$, owing to sharp gradients in $n(n>1)$, but the integral must always be positive.

The refractive index profile $r(n)$ follows from equation 11 and from the definition of $\eta$ (equation 3).

Transformation of the phase (5) follows similar lines, but involves slightly more manipulation. Applying the substitution (6), we find that the Abel pair ( 8 ) is again useful, but $g(y)$ is not apparent by inspection. We find:

$$
\begin{aligned}
& g^{\prime}(y)=\left(y+r_{0}^{2}\right) \frac{d \ln r}{d y} \\
& =\frac{d}{d y}\left[\frac{1}{K \pi} \int_{0}^{y} f(w)(w-y)^{-(1 / 2)} d w\right]
\end{aligned}
$$

$\eta^{2} \frac{d \ln r}{d \eta}$

$$
=\frac{d}{d \eta}\left[\frac{1}{k_{0} \pi} \int_{r_{0}}^{\eta} p \Phi(p)\left(p^{2}-\eta^{2}\right)^{-(1 / 2)} d p\right]
$$

Integration of (13) yields

$$
\begin{aligned}
& \ln \left(\frac{r}{r_{0}}\right)=-\frac{1}{k_{0} \pi} \int_{\eta}^{r_{0}} \frac{1}{u^{2}} \frac{d}{d u} \\
& \cdot\left[\int_{r_{0}}^{u} p \Phi(p)\left(p^{2}-u^{2}\right)^{-(1 / 2)} d p\right] d u
\end{aligned}
$$

and integration by parts transforms (14) to

$$
\begin{aligned}
& \ln \left(\frac{r}{r_{0}}\right) \\
& =-\frac{1}{k_{0} \pi}\left[\frac{1}{\eta^{2}} \int_{\eta}^{r_{0}} p \Phi(p)\left(p^{2}-\eta^{2}\right)^{-(1 / 2)} d p\right. \\
& \left.\quad-2 \int_{\eta}^{r_{0}} \frac{1}{u^{3}} \int_{u}^{r_{0}} p \Phi(p)\left(p^{2}-u^{2}\right)^{-(1 / 2)} d p d u\right]
\end{aligned}
$$

The second term can be simplified by interchanging the order of integration and integrating by parts:

$$
\begin{aligned}
& \ln \left(\frac{r}{r_{0}}\right)=-\frac{1}{\eta^{2} \frac{k_{0} \pi}{k_{0}}} \int_{\eta}^{r_{0}} p \Phi(p)\left[\left(p^{2}-\eta^{2}\right)^{-(1 / 2)}\right. \\
& \left.-\frac{1}{p^{2}}\left(p^{2}-\eta^{2}\right)^{1 / 2}-\frac{\eta^{2}}{p^{3}} \cosh ^{-1}\left(\frac{p}{\eta}\right)\right] d p \quad(16)
\end{aligned}
$$

This expression is a formal solution to the inversion problem using the phase as a data input. A simpler expression is obtainable by manipulating partial integrations to bring out a $\cosh ^{-1}$ factor in the first two terms:

$$
\begin{aligned}
r(\eta) & =r_{0} \exp \left[-\frac{1}{k_{0} \pi} \int_{r_{0}}^{\eta} \cosh ^{-1}\left(\frac{p}{\eta}\right) \frac{1}{p} \frac{d \Phi}{d p} d p\right] \\
& =r_{0} \exp (-N)
\end{aligned}
$$

By successfully solving the integral equation in the phase (5), we have demonstrated a method for determining the seismic velocitydepth function, using the travel time and the travel-time derivative $d T / d \Delta$ as inputs.

In both (17) and (11), one is effectively calculating the radius of maximum penetration of the ray with impact parameter $\eta$. Since it is desirable to work with small quantities where possible, we transform these expressions to a more useful form. Let $\theta_{u}$ and $\Phi_{u}$ be appropriate to a ray with the given impact parameter if the atmosphere is missing. Then define residuals $\theta_{a}$ and $\Phi_{a}$ :

$$
\begin{aligned}
& \theta=\theta_{u}+\theta_{a} \\
& \Phi=\Phi_{u}+\Phi_{a}
\end{aligned}
$$

Since both $M$ and $N$ operate linearly on the data, we may divide them into two terms in the same way, giving 


$$
\begin{aligned}
& r(\eta)=r_{0} \exp \left(-H_{u}\right) \exp \left(-M_{a}\right) \\
& r(\eta)=r_{0} \exp \left(-N_{u}\right) \exp \left(-N_{a}\right)
\end{aligned}
$$

If there is no atmosphere, $r_{n}(\eta)=\eta$, so that

$$
\begin{aligned}
& r(\eta)=\eta \exp \left(-M_{a}\right) \\
& r(\eta)=\eta \exp \left(-V_{a}\right)
\end{aligned}
$$

which give, independently, the refractive index profile in terms of an anomalous component of the data. It is to be emphasized that $\Phi$ and $\Phi_{w}$ refer to different rilys, with the samo impact parameter, for different atmospheres. $\Phi_{n}$ is a residual that is not aseociated with a single ray. It is also possible to choose, as a reference me, some standard anmopshere, for which $r_{n}(\eta)=$ $f(\eta)$ is known. The residual phise is computed with respect to this nominal phase, giving

$$
\begin{array}{r}
r(\eta)=f(\eta) \exp \left(-M_{a}\right) \\
=f(\eta) \exp \left(-N_{a}\right)
\end{array}
$$

Since an atmosphere is thin compared with the planetary radius, (19) maly be approximated by

$$
\Delta r=\eta-r \approx \eta N_{a}
$$

At the bottom of a putative 100-km Cytherean atmosphere, the total error would be less than $1 \mathrm{~km}$.

\section{Reduction of Doppler Data}

We now show how an impact parameter $p$ is associated with each ray; permitting use of one of the inversion formulas for the refractive index. Fjeldbo and Eshteman [1965] describe an approximation to determine the angle of the ray. This information is contained without ambiguity in the Doppler data in the following way.

The observed Doppler shift is corrected for the relative motion of the earth-based receiver and the planetary center of mass. The residual Doppler shift is then converted into a total phase by

$$
\phi(t)=\int_{t_{0}}^{t} \Delta f(t) d t
$$

where the zero of time is taken shortly before occultation. In the ray-optical description of the experiment, one can set up a family of constant phase surfaces and orthogonal rays.
The selection of a time zero provides a unique value to the phise function as a function of the spatial coordinates. The spacecraft trajectory provites a sampling of the phase along an arc. This information determines the angle of emission of the ray from the spacecraft and, eventually, the refractive index profile.

The eiconal equation for the phase is

$$
(\nabla \phi)^{2}=n^{2} f^{2} / c^{2}
$$

(Spaceratit phise is denoted by $\phi$. The phase along a curved atmospheric path referred to a top level of $r$, in denoted by $\Phi$.) Assuming the spacecraft is thove the atmosphere

$$
|\nabla \phi|=f / c
$$

From the Doppler datit, the directional derivative $\partial \phi / \partial s$ is determined along the spacecraft trajectory. The angle between the ray and the trajectory then hecomes

$$
\cos \psi=\frac{\partial \phi}{\partial s} /|\nabla \phi|=\frac{c}{f} \frac{\partial \phi}{\partial s}
$$

where $f$ may without error be taken as constant during the occultation. Since $\alpha+\psi$ is known, a follows immediately. Let the sulsecript $e$ refer to an cuperturbed ray, drawn as a straight line from spacecraft to earth. Then, setting time as the independent variable

$$
\frac{\partial \phi}{\partial s}=\frac{1}{\dot{s}} \frac{d \phi}{d t}=\frac{1}{\dot{s}}\left(\frac{d \phi_{e}}{d t}+\frac{d \phi_{n t m}}{d t}\right)
$$

The first term is due to the known relative motion in planetocentric coordinates, and the second is due to the atmosphere. Let $D$ signify an instantaneous Doppler shift; then

$$
\frac{\partial \phi}{\partial s}=\frac{1}{\dot{s}}\left(D_{e}+D_{\mathrm{at} t \mathrm{~m}}\right)=\frac{D}{\dot{s}}
$$

where $D_{\text {at } m}$ is the residual normally used in discussions of the atmosphere [Kliore et al., 1966]

$$
\cos \psi=\frac{c}{f \dot{s}}\left(D_{e}+D_{\mathrm{utm}}\right)
$$

Now

$$
\cos \psi_{e}=\frac{c}{f \dot{s}} D_{e}
$$

so that the deviation angle is 


\section{$\tan \frac{1}{2}\left(\psi-\psi_{\bullet}\right)$}

$$
=\frac{-D_{\mathrm{atm}}}{\left[D_{s}^{2}-\left(D_{e}+D_{\mathrm{atm}}\right)^{2}\right]^{1 / 2}+\left[D_{s}^{2}-D_{e}{ }^{2}\right]}
$$

where $D_{s} \equiv(f \dot{s} / c)$, and $D_{s} \geqq D_{e}$. Equation 28 is general, but it can normally be approximated. When $\psi-\psi_{c} \ll 1, D_{\mathrm{atm}} \ll D_{s}, D_{\mathrm{atm}} \ll D_{e}$, then to first order

$$
\psi-\psi_{e}=\Delta \psi \approx \frac{-D_{x+m}}{\left(D_{s}{ }^{2}-D_{e}{ }^{2}\right)^{1 / 2}}
$$

which applies as long as $D_{s}$ is not close to $D_{e}$.

The impact parameter follows, by application of $(28)$ :

$$
p=r_{s} \sin \alpha=r_{s} \sin \left(\alpha_{e}+\Delta \psi\right)
$$

when the approximation (29) is suitable,

$$
p-p_{e}=\Delta p \approx \Delta \psi\left(r_{s}{ }^{2}-p_{e}{ }^{2}\right)^{1 / 2}
$$

The values of $\Phi$ and $\Phi_{u}$ or of $\theta$ and $\theta_{u}$ are needed. The ray is projected to a reference sphere $r_{0}$, which lies sensibly above the atmosphere. The angle of incidence at this surface is

$$
\sin i_{0}=\left(r_{s} \sin \alpha\right) / r_{0}
$$

(the subscript $s$ refers to spacecraft coordinates). Solution of the triangles in Figure 1 gives the entry point angle

$$
\begin{aligned}
\sin \left(\Omega_{0}\right. & \left.-\Omega_{s}\right) \\
& =\frac{\sin \alpha}{r_{0}}\left(r_{s} \cos \alpha-r_{0} \cos i_{0}\right)
\end{aligned}
$$

and the angle subtended by the portion of the ray in the atmosphere is

$$
\theta=\pi-i_{0}{ }^{\prime}-\Omega_{0}
$$

The rays to earth are sufficiently parallel that we can take $i_{v}^{\prime}=i_{p}^{\prime}$. $\theta_{u}=2 \cos ^{-1}\left(p / r_{b}\right)$ follows, by inspection of the figure.

Before reducing the observed phase to a function appropriate for input to (19) or (21), we transform the expression for $N_{u}$. Using time as an independent variable

$$
N_{a}(t)=\frac{1}{k_{0} \pi} \int_{0}^{t} \cosh ^{-1}\left[\frac{p(\tau)}{p(t)}\right] \frac{1}{p(\tau)} D_{a} d \tau
$$

where

$$
D_{a}=d \Phi_{a} / d \tau .
$$

The natural time origin for the measured phase $\phi$ is at occultation by $r=r_{0}$ when $p=r_{0}$. Then the atmospheric phase shift $\Phi$ is derived from $\phi$ by (Figure 2):

$$
\Phi=\phi-\phi_{s s^{\prime}}+\phi_{E E^{\prime}}+\phi_{A A^{\prime}}
$$

where the last, time-independent term is added to make $\Phi=0$ at $p=r_{0}$. Now, $\Phi_{a}=\Phi-$ $\Phi_{u}$, hence

$$
\Phi_{a}=\phi-\phi_{s s^{\prime}}+\phi_{E E^{\prime}}+\phi_{A A^{\prime}}-\Phi_{u}
$$

where

$$
\begin{aligned}
\phi_{E E^{\prime}} & =k_{0} r_{0} \cos i_{0} \\
\phi_{s s^{\prime}} & =k_{0}\left[r_{s} \cos \alpha-r_{0} \cos i_{i^{\prime}}\right] \\
\phi_{A A^{\prime}} & =k_{0} r_{* 0} \cos \alpha_{0} \\
\Phi_{u} & =2 k_{0} r_{0} \cos i_{0}
\end{aligned}
$$

giving

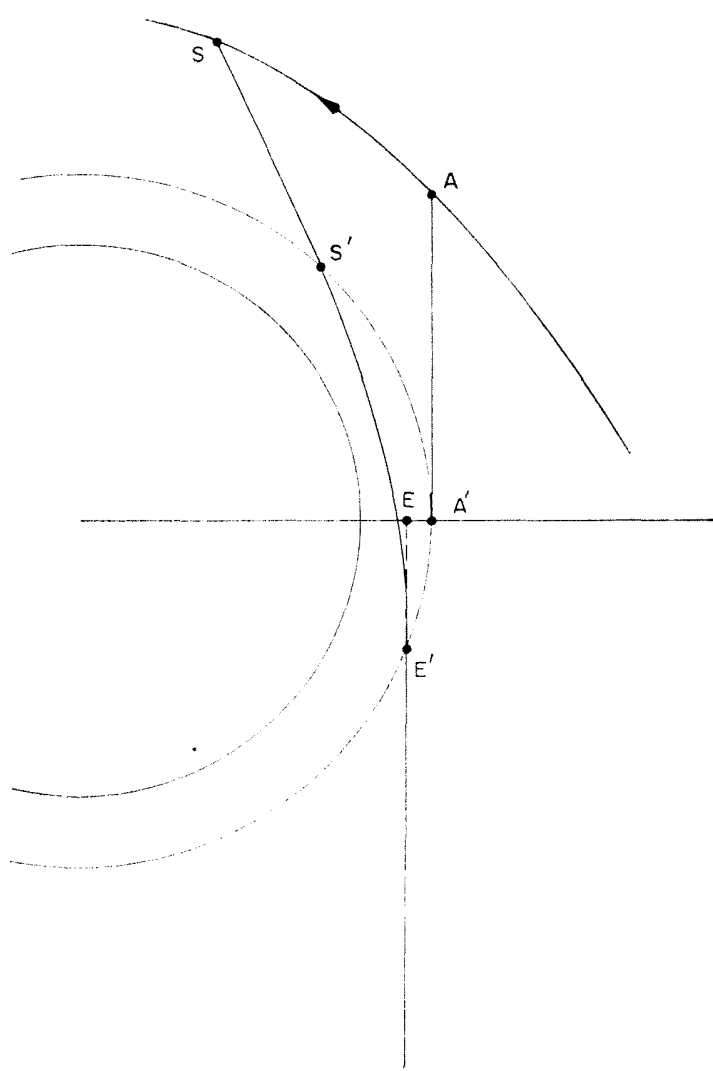

Fig. 2. Geometry for conversion of observed phase to atmospheric phase. 


$$
\begin{aligned}
\Phi_{a} & =\phi-k_{0}\left[r_{s} \cos \alpha-r_{s 0} \cos \alpha_{0}\right] \\
& =\phi-k_{0}\left[S S^{\prime \prime}-A A^{\prime}\right]=\phi-\phi_{c} .
\end{aligned}
$$

(see Figure 3). The reduced Doppler function $D_{a}$, in equation 36 , is thus obtained from the data by

$$
D_{a}=D-D_{c}=D-\frac{d \phi_{c}}{d \tau}
$$

In summary of this section, we find that the Doppler residual $D_{\mathrm{atm}}$ (equation 27) is used to determine the deflection of the ray and, consequently, its impact parameter. The Doppler residual $D_{a}$ (equation 38 ) is used in conjunction with the inversion formula 35 to determine the refractive index profile.

\section{Approximation of the Ray Integrals}

We now discuss the integrals (4) and (5), for $\theta$ and $\Phi$ in terms of refractivity variations. An expansion parameter is chosen that provides a perturbation expansion when the ray curvature is slight, and which causes the integrals to diverge, as required when the ray curvature at the bottom of the ray is equal to the curvature of a level surface.

Set

$$
\begin{aligned}
n & =1+\epsilon \\
& =n_{p}\left[1-\frac{\gamma_{p} g_{p}(r)}{r}\left(r-r_{p}\right)\right]
\end{aligned}
$$

where

$$
\gamma_{p} \equiv-\frac{r_{p}}{n_{p}}\left(\frac{d \epsilon}{d r}\right)_{r=r_{p}}
$$

is the appropriate expansion parameter. From the definition of ray curvature $1 / \rho$, we can show that

$$
\gamma_{p}=\frac{r_{p}}{\rho}
$$

The function $g_{p}(r)$ is used to absorb the remaining variability of $n$ with $r$. It is considered to be continuously differentiable. At the limits

$$
\begin{aligned}
& g_{p}\left(r_{0}\right)=0 \\
& g_{p}\left(r_{p}\right)=1
\end{aligned}
$$

(For brevity, we will write $g_{p}(r)$ as $g_{p}$.)

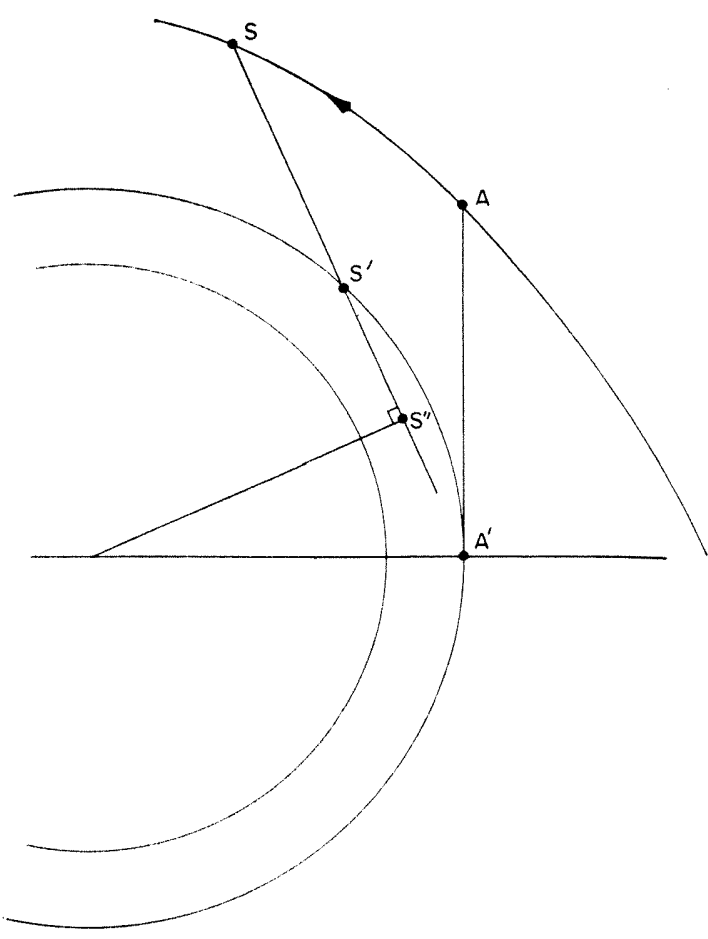

Fig. 3. Geometrical interpretation of phase conversion (37).

Now we write out the integrals (4) and (5) in terms of (39). Without approximation,

$$
\begin{aligned}
\theta= & 2 \int_{r_{p}}^{r_{0}} \frac{r_{p}}{r}\left(r^{2}-r_{p}^{2}\right)^{-(1 / 2)}\left\{1-\frac{2 \gamma_{p} g_{p} r}{r+r_{p}}\right. \\
& \left.\cdot\left[1-\frac{1}{2} \frac{\gamma_{p} g_{p}}{r}\left(r-r_{p}\right)\right]\right\}^{-(1 / 2)} d r
\end{aligned}
$$

and

$$
\begin{aligned}
\Phi= & 2 k_{0} n_{p} \int_{r_{p}}^{r_{0}}\left[1-\frac{\gamma_{p} g_{p}\left(r-r_{p}\right)}{r}\right]^{2} \\
& \cdot\left[r-r_{p}^{2}\right]^{-(1 / 2)}\left\{1-\frac{2 \gamma_{p} g_{p} r}{r+r_{p}}\right. \\
& \left.\cdot\left[1-\frac{1}{2} \frac{\gamma_{p} g_{p}}{r}\left(r-r_{p}\right)\right]\right\}^{-(1 / 2)} d r
\end{aligned}
$$

If $\gamma_{p}=1$, then the integrals will diverge, owing to the vanishing of the argument of the second square root as $r \rightarrow r_{p}$.

By assuming a thin atmosphere $\left[\left(r-r_{p}\right) \ll\right.$ $\left.r_{p}\right],(41)$ and (42) reduce to 
$\theta=2 \int_{r_{p}}^{r_{0}}\left(r^{2}-r_{p}^{2}\right)^{-(1 / 2)}$

$$
\cdot\left[1-\gamma_{p} g_{p}\right]^{-(1 / 2)} d r
$$

$\Phi=2 k_{0} n_{p} \int_{r_{\nu}}^{r_{0}}\left(r^{2}-r_{\nu}{ }^{2}\right)^{-(1 / 2)}$

$$
\cdot\left[1-\gamma_{\nu} g_{p}\right]^{-(1 / 2)} d r
$$

both of which diverge, as appropriate, at $\gamma_{n}=1$. In this approximation,

$$
\frac{\Phi}{\theta} \approx \frac{r_{p}}{\lambda_{p}} \approx \frac{r_{m}}{\lambda_{u}}=k_{v} r_{m}
$$

The fractional error in (45) will be about $\left(r_{0}-r_{p}\right) / r_{p}$ and can be made to vanish by an appropriate choice of the 'mean radius' $r_{m}$. The atmospheric perturbations in $\theta$ and $\Phi$ are normally small compared with the nominal values: it is not obvious whether this approximation, in general, will preserve the perturbations with the desired fractional error.

If (45) is valid, we can base an inversion procedure on (11) with the phase as input:

$r(\eta)=r_{0} \exp \left[-\frac{1}{\pi k_{0} r_{m}} \int_{r_{0}}^{\eta} \cosh ^{-1}\left(\frac{p}{\eta}\right) \frac{d \Phi}{d p} d p\right]$

A thin atmosphere approximation applied to (17) is $p \approx r_{m}$. We can thus obtain (46) directly from the phase inversion formula.

The approximation of a 'thin, weak' atmosphere is of some interest. It may be assumed in (43) and (44) that $\gamma_{p} \ll 1$. The square root may be expanded in power series in $\gamma_{p}$, and the resulting expressions form a perturbation series for the functions of interest:

$$
\begin{aligned}
\theta & =\theta_{0}+\theta_{1}+\cdots \\
\theta_{0} & =2 \cos ^{-1}\left(\frac{r_{p}}{r_{0}}\right) \\
\theta_{1} & =\gamma_{p} \int_{r_{p}}^{r_{0}} \frac{r_{p}}{r}\left(r^{2}-r_{p}^{2}\right)^{-(1 / 2)} g_{p}(r) d r \\
\Phi & =\Phi_{0}+\Phi_{1}+\cdots \\
\Phi_{0} & =2 k_{0}\left(r_{0}^{2}-r_{p}^{2}\right)^{-(1 / 2)} \\
\Phi_{1} & =k_{0} \gamma_{\nu} \int_{r_{p}}^{r_{0}} r\left(r^{2}-r_{p}^{2}\right)^{-(1 / 2)} g_{p}(r) d r
\end{aligned}
$$

In the lower atmosphere of a planet like Venus, (50) may not be satisfied, and rays with $p$ below a critical value can emerge from the atmosphere only by reflection from the solid surface. The essence of this, or any equivalent inversion method, is that the turning points $\left(r_{p}\right)$ of the rays considered must range continuously through the atmosphere. Since this is true only from $r_{0}$ down to the critical level, (11) or (17) cannot be applied to the atmosphere below the critical level. In seismology, refractive index decreases at great depth results in the eventual emergence of the downward refracted rays, but this is extremely unlikely in atmospheric studies.

A slightly different condition defines when a ray having the accessible range of $p, 0<p<$ $r_{0}$, can have a turning point at depth $r$ :

$$
n r<r_{0}
$$

If a screening region of low index (ionosphere) lies above the neutral atmosphere, equation 14 is replaced by the more stringent condition. 


$$
n r<n^{*} r^{*}
$$

where * refers to the ionospheric level of maximum $n r$. For an ionosphere with the scale determined for Mars by Mariner 4 , about $6 \times 10^{3}$ electrons $/ \mathrm{cm}^{3}$ would be required. Consequently, equation 52 is unlikely to be of concern in a real case.

The intensity variation of the refracted signal due to focusing and defocusing can be stated in the notation of this paper. Neglecting diffraction effects at caustics,

$\frac{I \text { (exit) }}{I(\text { entry })}=\frac{\sin \Theta}{\sin (\theta+\Theta)}\left[\frac{d \theta / d p}{\left\{\left[\left(\tan i_{0}-\tan \alpha\right) \sin \alpha / r_{0} \cos \Theta\right]+\left(\tan \Theta / r_{0} \sin i_{0}\right)\right\}}-1\right]^{-1}$

where $\Theta=\Omega_{0}-\Omega_{s}$. If $r_{s}$ is nearly equal to $r_{0}$, the expression in braces is small and positive; $I$ (exit) will be very large (focusing) if $d \theta / d p=$ $0+\{\} \rightarrow 0$. If $r_{s} \rightarrow \infty, \alpha \rightarrow 0, \Theta \rightarrow i_{0}$, and focusing will occur if $d \theta / d p=\tan i_{0}$. In the study of atmospheres, $i_{0}$ is always near $\pi / 2$, and that condition will not be satisfied (Figure 4 ).

Rays that are refracted downward at nearly the curvature of the planet have large $d \theta / d p$ and are severely defocused.

\section{Appendix}

In solving the integral equation 5 , we have demonstrated an approach to the solution of the general integral equation

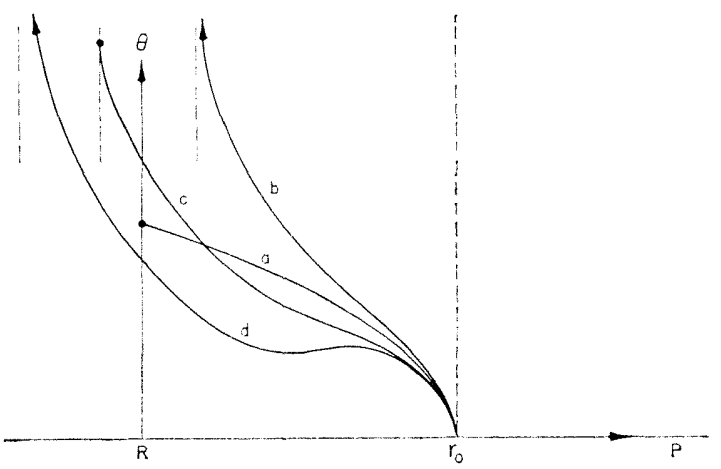

Fig. 4. Schematic $\theta(p)$ curves for different atmospheres: (a) no atmosphere: $\theta=2 \cos ^{-1}\left(p / r_{0}\right)$; $p>R ;(b)$ strongly refracting neutral atmosphere; (c) weakly refracting ionosphere and weakly refracting atmosphere; $(d)$ strongly refracting ionosphere and strongly refracting atmosphere. (a) and (c) end by occultation; (b) and $(d)$ end asymptotically at an optical shadow.

$$
f(w)=-K \int_{0}^{w} \frac{1}{\lambda(y)} g^{\prime}(y)(y-w)^{-(1 / 2)} d y
$$

where $1 / \lambda(y)$ is a weighting function. Carrying the analysis to the stage of (15) gives a formal solution:

$$
\begin{aligned}
g(y)=\frac{1}{\pi K} & \left\{\int _ { 0 } ^ { y } f ( w ) \left[\lambda(y)(w-y)^{-(1 / 2)}\right.\right. \\
& \left.\left.-\int_{w}^{y} \lambda^{\prime}(\eta)(w-\eta)^{-(1 / 2)} d \eta\right] d w\right\}
\end{aligned}
$$

Acknowledgments. The authors are indebted to $G$. Fjeldbo for his discussion of the problem with the 'TYCHO' group. We wish to thank A. J. Kliore for his comments on the manuscript. This research was performed as part of the 1967 'TYCHO' Summer Study at Dartmonth College under NASA contract NSR-24-005-047 with the University of Minnesota.

\section{References}

Bateman, H., The solution of the integral equation which connects the velocity of propagation of an earthquake wave in the interior of the earth with the times which the disturbance takes to travel to the different stations on the earth's surface, Phil. Mag. 19, 576, 1910.

Bullen, K. E., An Introduction to the Theory of Seismology, Cambridge University Press, New York, 1963.

Cain, D. L., F. D. Drake, V. R. Eshleman, G. Fjeldbo, A. Kliore, and G. S. Levy, Proc. Ionospheric Research Committee of the Avionics Panel, AGARD, NATO, Rome, Italy, September 21-25, 1965.

Cain, D. L., A. J. Kliore, and G. S. Levy, The Mariner 4 occultation experiment: Summary of data and deduction methods, AIAA preprint No. 66-148, 1966.

Fjeldbo, G., and V. R. Eshleman, The bistatic radar-occultation method for the study of planetary atmospheres, J. Geophys. Res., 70, 3217, 1965.

Fjeldbo, G., V. R. Eshleman, A. J. Kliore, D. L. Cain, G. S. Levy, and F. D. Drake, Preliminary results of the Mariner 4 radio occultation measurement of the upper atmosphere of Mars, Proc. Caltech-JPL Lunar and Planetary Conf., Pasadena, California, September 13-18, $1965 b$.

Fjeldbo, G., W. C. Fjeldbo, and V. R. Eshleman, Models for the atmosphere of Mars based on the Mariner 4 occultation experiment, J. Geophys. Res., $71,2307,1966 a$. 
Fjeldbo, G., W. C. Fjeldbo, and V. R. Eshleman, Atmosphere of Mars: Mariner 4 models conpared, Science, 153, 1518, $1966 b$.

Herglotz, G., Uber das Benndorfsche Problem der Fortpflanzungsgeschwindigkeit, der Erdbebenstrahlen, Phys. Z., S, 145, 1907.

Jeffreys, H., The Earth, Cambridge University Press, New York, 1959.

Kliore, A., D. L. Cain, and G. S. Lery, Radio occultation measurement of the Martian atmosphere over two regions by the Mariner 4 space probe, Proc. Scventh Intern. Space Sci. Symp. (COSPAR), Vienna, Austria, May 11-17, 226, 1966.

Kliore, A., D. L. Cain, G. S. Levy, V. R. Eshleman,
G. Fjeldbo, and F. D. Drake, Preliminary results of the Mariner 4 occultation measurement of the atmosphere of Mars, Proc. Caltech-JPL Lunar and Planetary Conf., September 13-18, 57, $1965 a$.

Kliore, A., D. L. Cain, G. S. Levy, V. R. Eshleman, G. Fjeldbo, and F. D. Drake, Occultation experiment: Results of the first direct measurement of Mars' atmosphere and ionosphere, Science, $1.49,1243,1965 b$.

Wiechert, E., and I. Geiger, Bestimmung des Weges der Erdbebenwellen im Erdinnern, Phys. Z., 11, 294, 1910.

(Received September 5, 1967.) 